\title{
A REMARK ON EXPANDING MAPS
}

\author{
KUNG-CHING CHANG AND LI SHUJE
}

\begin{abstract}
In this paper we discuss the following problem stated by $L$. Nirenberg: Let $T$ be an expanding map $H \rightarrow H$ ( $H$ is a Hilbert space) with $T(0)=0$. Suppose $T$ maps a neighborhood of the origin onto a neighborhood of the origin. Does $T$ map $H$ onto $H$ ?

We answer positively the problem when $T$ is differentiable.
\end{abstract}

In [1] L. Nirenberg stated the following open problem:

Suppose $T$ is a continuous map $H \rightarrow H$ ( $H$ is a Hilbert space) which is expanding, i.e. $\|T x-T y\| \geq\|x-y\|$, and $T(0)=0$. Suppose $T$ maps a neighborhood of the origin onto a neighborhood of the origin. Does $T$ maps $H$ onto $H$ ? If we consider $\alpha T$ instead of $T$ for a real $\alpha>1$, with no loss of generality, we may assume that $\|T x-T y\| \geq \alpha\|x-y\|$.

When $H$ is a Euclidean space $R^{n}$, we know that it is true because of the Domain Invariance Theorem (in this case the condition that $T$ maps a neighborhood of the origin onto a neighborhood of the origin can be omitted). When $H$ is a Hilbert space there is a Domain Invariance Theorem for the following cases:

(i) $T=I-C$ where $I$ is the identity and $C$ is a compact operator, or

(ii) $T$ is a strongly monotone operator.

(In case (i) see [2], in case (ii) see [3].) But, in general, the Domain Invariance Theorem does not hold; a simple counterexample is when $T$ is the shift operator.

In this paper we answer positively the problem when $T$ is differentiable.

We express our gratitude to Professor Louis Nirenberg and Dr. Brian Rowley for their useful suggestions.

LEMMA 1. Suppose $T$ is an expanding map from a Banach space $X$ into a Banach space $Y, T(0)=0, T$ maps a neighborhood $N_{x}(0)$ of the origin of $X$ onto a neighborhood $N_{Y}(0)$ of the origin of $Y$, and $T$ is Fréchet-differentiable at the origin of $X$, then $\left[T^{\prime}(0)\right]^{-1}$ exists and $\left\|\left[T^{\prime}(0)\right]^{-1}\right\| \leq 1 / \alpha<1$.

Proof. First we prove $R T^{\prime}(0)$ (range of $T^{\prime}(0)$ ) is dense in $Y$. If the statement of the lemma is not true, there must exist a $z_{0} \in Y^{*}\left(Y^{*}\right.$ is the conjugate space) such that $z_{0}\left(T^{\prime}(0) x\right)=0 \forall x \in X$.

Set

$$
T(x)=T(0)+T^{\prime}(0) x+0(\|x\|),
$$

we have

$$
z_{0}(T(x))=z_{0}\left(T(0)+T^{\prime}(0) x\right)+z_{0}(0(\|x\|))
$$

Received by the editors December 1, 1979 and, in revised form, June 17, 1981.

1980 Mathematics Subject Classification. Primary 47H15.

Key words and phrases. Expanding map, Hadamard implicit function theorem, potential operator. 
There exists a $\bar{z}_{0} \in Y$ such that $z_{0}\left(\bar{z}_{0}\right)=\left\|\bar{z}_{0}\right\|\left\|z_{0}\right\|_{*}$. We know that $T$ maps $N_{x}(0)$ onto $N_{Y}(0)$ from assumption, so that there exists $\left\{x_{n}\right\} \subset N_{x}(0)$ such that $T x_{n}=$ $\alpha_{n} \bar{z}_{0}$. By $\left\|T x_{n}\right\|>\left\|x_{n}\right\|$ and $\alpha_{n} \rightarrow 0$ we find $x_{n} \rightarrow 0$ and

$$
\left|z_{0}\left(T\left(x_{n}\right)\right)\right|=\left|z_{0}\left(\alpha_{n} \bar{z}_{0}\right)\right|=\left\|\alpha_{n} \bar{z}_{0}\right\|\left\|z_{0}\right\|_{*}=\left\|T x_{n}\right\|\left\|z_{0}\right\|_{*} \geq\left\|x_{n}\right\|\left\|z_{0}\right\|_{*} .
$$

On the other hand

(2) $\left|z_{0}\left(T\left(x_{n}\right)\right)\right|=\left|z_{0}\left(T^{\prime}(0) x_{n}\right)+z_{0}\left(0\left(\left\|x_{n}\right\|\right)\right)\right|=\left|z_{0}\left(0\left(\left\|x_{n}\right\|\right)\right)\right| \leq\left\|z_{0}\right\|_{*}\left\|0\left(\left\|x_{n}\right\|\right)\right\|$.

By (1) and (2) we have

$$
\left\|x_{n}\right\| \leq\left\|0\left(\left\|x_{n}\right\|\right)\right\| \quad \text { as } x_{n} \rightarrow 0
$$

This contradiction shows $R T^{\prime}(0)$ is dense in $Y$.

Second we show $R T^{\prime}(0)=Y$. It is sufficient to prove $R T^{\prime}(0)$ is a closed set in $Y$.

Suppose $T^{\prime}(0) x_{n}=y_{n} \rightarrow y$. We know that there exists the inverse of $T^{\prime}(0)$ on $R T^{\prime}(0)$. Since $T$ is expansive therefore $\left\|\left[T^{\prime}(0)\right]^{-1}\right\| \leq 1 / \alpha<1$ on $R T^{\prime}(0)$ and $x_{n}=\left[T^{\prime}(0)\right]^{-1} y_{n},\left\|x_{n}-x_{m}\right\| \leq\left\|\left[T^{\prime}(0)\right]^{-1} \mid\right\|\left\|y_{n}-y_{m}\right\| \rightarrow 0$, so that there exists $x \in X$ such that $x_{n} \rightarrow x$ and $T^{\prime}(0) x=y$, this fact show $\left[T^{\prime}(0)\right]^{-1}$ exists and $\left\|\left[T^{\prime}(0)\right]^{-1}\right\|<1$ on $Y$. Q.E.D.

The following Lemma 2 is an immediate corollary of the Theorem 2 in [4]. We only need the following special case in [4].

Proposition 1. Suppose $X$ and $Y$ are real Banach spaces, $f$ is a map from $X$ into $Y, f$ has a linear Gâteaux differential $f^{\prime}(x)$, a bounded linear operator, at every point $x \in X$, and $N\left(\left[f^{\prime}(x)\right]^{*}\right)=0 \forall x \in X$, where $N\left(\left[f^{\prime}(x)\right]^{*}\right)$ denotes the null space of $\left[f^{\prime}(x)^{*}\right]$ and $\left[f^{\prime}(x)\right]^{*}$ denotes the adjoint operator of $\left[f^{\prime}(x)\right]$. If $f(X)$ is closed in $Y$ then $f(X)=Y$.

LEMMA 2. Suppose $X$ and $Y$ are real Banach spaces, $T$ is a map from $X$ into $Y$ with closed $T(X), T$ has a linear Gâteaux differential $T^{\prime}(x)$, a bounded linear operator, at every point $x \in H$. If $\left[T^{\prime}(x)\right]^{-1}$ exists $\forall x \in X$ then $T(X)=Y$.

ProOF. By Proposition 1 it is sufficient to prove $N\left[T^{\prime}(x)^{*}\right]=0 \forall x \in X$. If it is not true then there must exist a $x_{0} \in X$ such that $N\left(T^{\prime}\left(x_{0}\right)^{*}\right) \neq 0$, so that there exists $y_{0}^{*} \neq 0, y_{0}^{*} \in Y^{*}$ such that $\left[T^{\prime}\left(x_{0}\right)\right]^{*} y_{0}^{*}(x)=0$, i.e. $y_{0}^{*}\left(T^{\prime}\left(x_{0}\right)\right)(x)=0$ but $R T^{\prime}\left(x_{0}\right)=Y$ therefore we have $y_{0}^{*}=0$ this is a contradiction. Q.E.D.

LEMMA 3. Suppose $T_{1}, T_{2}$ are linear operators and $T_{1}$ has a bounded inverse $T_{1}^{-1}$ with $\left\|T_{1}^{-1}\right\|<1$. If $\left\|T_{1}-T_{2}\right\|<1$ holds, then $T_{2}$ has a bounded inverse $T_{2}^{-1}$.

ProOF. We know $T_{2}=T_{1}\left(I+\left(T_{2}-T_{1}\right) T_{1}^{-1}\right)$, since $\left\|\left(T_{2}-T_{1}\right) T_{1}^{-1}\right\| \leq$ $\left\|T_{2}-T_{1}\right\|\left\|T_{1}^{-1}\right\|<1$, so that $\left[I+\left(T_{2}-T_{1}\right) T_{1}^{-1}\right]^{-1}$ exists and $T_{2}$ has a bounded inverse. Q.E.D.

THEOREM 1. Suppose $X$ is a real Banach space and $Y$ is a Banach space, also suppose $T$ is a expanding map from $X$ into $Y, T$ is Fréchet-differentiable in $X$ and $\forall x_{0} \in X$ we have $\varlimsup_{x \rightarrow x_{0}}\left\|T^{\prime}(x)-T^{\prime}\left(x_{0}\right)\right\|<1, T(0)=0$ and $T$ maps a neighborhood of the origin of $X$ onto a neighborhood of the origin of $Y$. Then $T X=Y$. 
Proof. Firstly, it is easy to show that the image of any expanding map is closed. If the statement of the theorem is not true then by Lemmas 2 and 3, we know that there exists a nonempty closed set $S \subset X$, such that $\left[T^{\prime}(x)\right]$ does not have an inverse $\forall x \in S$. From Lemmas 1,3 and the assumption of the theorem, we have a neighborhood of the origin, which does not intersect with $S$. Therefore there exists a ray intersecting with $S$, say $\left\{t \bar{x} \mid t \in \mathbf{R}_{+}^{\prime}\right\}$. Then there is a $r>0$ such that $t \bar{x} \in S$ for $t \in[0, r)$ and $r \bar{x} \in S$. Let $x_{n}=(r-1 / n) \bar{x}$, we have $x_{n} \notin S$ i.e. $\left[T^{\prime}\left(x_{n}\right)\right]^{-1} \exists$, and we have $\left\|\left[T^{\prime}\left(x_{n}\right)\right]^{-1}\right\| \leq 1 / \alpha<1$. Again, by assumption

$$
\varlimsup_{x_{n} \rightarrow r \bar{x}}\left\|T^{\prime}\left(x_{n}\right)-T^{\prime}(r \bar{x})\right\|<1 \text {, }
$$

and by Lemma 3 we know that $\left[T^{\prime}(r \bar{x})\right]^{-1}$ exist, which contradicts $r \bar{x} \in S$. Q.E.D.

REMARK 1. In particular when $T \in C^{1}$ Theorem 1 is true, we could prove it by the open and closed mapping argument instead of Lemma 2.

REMARK 2. Suppose $X=Y=H$ is a Hilbert space, $T$ is an expanding map and has a linear Gâteaux differential $T^{\prime}(x)$ at every point $x \in H$, which is selfadjoint. Then $T$ maps $H$ onto $H$. We could find this conclusion from the fact that the residual spectrum of a selfadjoint operator is empty.

REMARK 3. In Theorem 1 if $T$ satisfies

$$
\|T x-T y\| \geq \alpha\|x-y\| \quad \forall x, y \in X,
$$

where $\alpha>0$ and if

$$
\varlimsup_{x \rightarrow x_{0}}\left\|T^{\prime}(x)-T^{\prime}\left(x_{0}\right)\right\|<\alpha \quad \forall x_{0} \in X
$$

Then $T X=Y$.

The following Proposition 2 is the Theorem 5.1 in [5].

Proposition 2. Suppose $X$ is a Banach space, $F$ is an operator from $X$ into the conjugative space $X^{*}, F$ has a linear Gâteaux differential $D F(x, h)$ at every point of the ball $B\left(x_{0}, r\right)$. The functional $\left(D F\left(X, h_{1}\right), h_{2}\right)$ is continuous in $x$ at every point of $B\left(x_{0}, r\right)$, the operator $F$ is potential in $B\left(x_{0}, r\right)$, then the bilinear functional $\left(D f\left(x, h_{1}\right), h_{2}\right)$ is symmetric for every $x \in B\left(x_{0}, r\right)$.

By Proposition 2 we have the following result:

THEOREM 2. Suppose $T$ is an expanding map from $H$ into $H, T \in C^{1}$ and $T$ is a potential operator, then $T$ maps $H$ onto $H$.

Proof. For any $x_{0} \in H$ there is a ball $B\left(x_{0}, r\right)$ such that in which the conditions in Proposition 2 are satisfied. Therefore $\left(D T\left(x, h_{1}\right), h_{2}\right)$ is symmetric for every $x \in$ $B\left(x_{0}, r\right)$ and since $T$ is Fréchet-differentiable we know that $T^{\prime}(x)$ is a selfadjoint operator $\forall x \in H$. By Remark 2 we have $T H=H$. Q.E.D.

The referee has pointed out that Theorem 2 extends to a reflexive Banach space.

\section{REFERENCES}

1. L. Nirenberg, Topics in nonlinear functional analysis, Lecture Notes, Courant Inst., New York Univ., New York, 1974.

2. J. T. Schwartz, Nonlinear functional analysis, Gordon and Breach, New York, 1969.

3. G. J. Minty, Monotone (nonlinear) operators in Hilbert space, Duke Math. J. 29 (1962). 
4. F. Browder, On the Fredholm alternative for nonlinear operators, Bull. Amer. Math. Soc. 76 (1970), 993-998.

5. M. M. Vainberg, Variational methods for the study of nonlinear operators, Holden-Day, San Francisco, Calif., 1964.

Department of Mathematics, Peking University, Peking, China

Courant Institute of Mathematical SCIENCES, NeW YORK UNIVERsity, NeW YORK, NEW YORK 10012

institute of Mathematics, ACademia Sinica, Peking, China 